\title{
Searches for hadronically decaying Dark Matter mediator particles at ATLAS
}

\author{
Herjuno Nindhito* \\ Lund University (SE) \\ E-mail: herjuno.rah.nindhitodcern.ch
}

\begin{abstract}
Searches for hadronic resonances of the Dark Matter (DM) particles in the sub-TeV mass region remain as a viable target at ATLAS. However, due to the bandwidth limitation, the events that available for performing an analysis were statistically limited. Reducing the event size by recording a fraction of the full event information overcomes this limitation. An analysis that is performed on those events is called Trigger-Level Analysis(TLA). This poster highlights the TLA strategy used to search for low-mass dijet resonances. No significant excesses are found in a region between 450 and $950 \mathrm{GeV}$. As an addition, limits are set on a simplified leptophobic Z' model of DM mediator with axial coupling to quarks and DM particles as well as on Gaussian resonances.
\end{abstract}

Fourth Annual Large Hadron Collider Physics

13-18 June 2016

Lund, Sweden

* Speaker.

${ }^{\dagger}$ On behalf of the ATLAS collaboration 


\section{Introduction}

A search for DM mediator particles via dijet resonance in the sub-TeV region benefits greatly from the high luminosity run in ATLAS Run-2[1]. However, a trigger prescaling needs to be applied to that region due to a bandwidth limitation. As a consequence, numerous potential events are discarded, limiting events availability for this kind of search.

With fixed bandwidth allocation, recording only a subset of full jet information will improve event rate considerably. Limited information in these events is partially-reconstructed and calibrated to be used later on in the Trigger-Level Analysis (TLA).

\section{Trigger-level analysis}

Trigger-Level Analysis (TLA) is the usage of the High Level Trigger (HLT) objects which collected via Data Scouting (DS) technique to perform a physics analysis. Figure 1 shows the DS

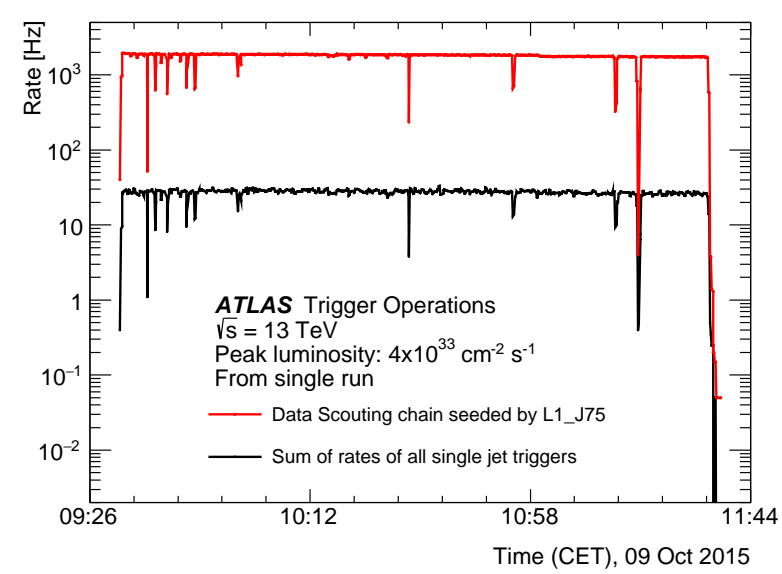

Figure 1: Trigger rate for the data scouting chain seeded by the L1_J75 compared to the sum of the rates of all central single jet triggers, plotted against a time range of a single run. DS chain have more statistics due to smaller event size recorded.

stream has higher rates compared to the sum of single trigger rates.

\section{High level trigger calibration and performance}

Jets, as a collection of particles, which reconstructed at the trigger level are calibrated to be as close as possible to jets in the particle level. Since DS stream does not save tracking information, calibration procedures such as origin correction and Global Sequential Correction (GSC)[2] which require tracking are not applied. Figure 2 a shows dijet invariant mass of leading and sub-leading HLT jets compared to its offline counterpart in data. Black line indicates the average $m_{j j}$ response between HLT and offline jets. It is shown that the difference is within $1 \%$ of the unity value. Additional $\eta$ inter calibration is also performed to correct for detector dependence effect as shown in Figure 2b. 


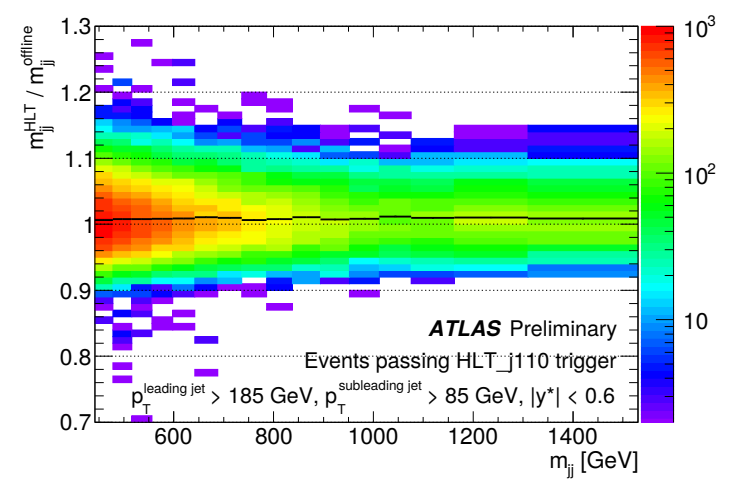

(a)

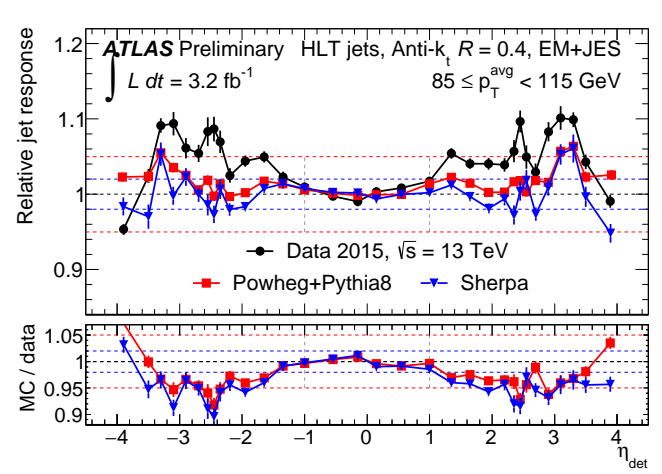

(b)

Figure 2: Dijet mass response comparison of HLT and offline jets passed HLT_j110 (a) and centralforward calibration factors in data and $\mathrm{MC}(\mathrm{b})$

\section{Search phase and limit setting}

The search is performed by selecting events with at least two jets event with $\eta<2.8$. The minimum leading jet $p_{T}$ is $185 \mathrm{GeV}$, with sub-leading jet should have $85 \mathrm{GeV}$ of transverse momentum as a minimum value. Two signal regions then defined as $y^{*}<0.6$ and $y^{*}<0.3$. Figure $3 a$

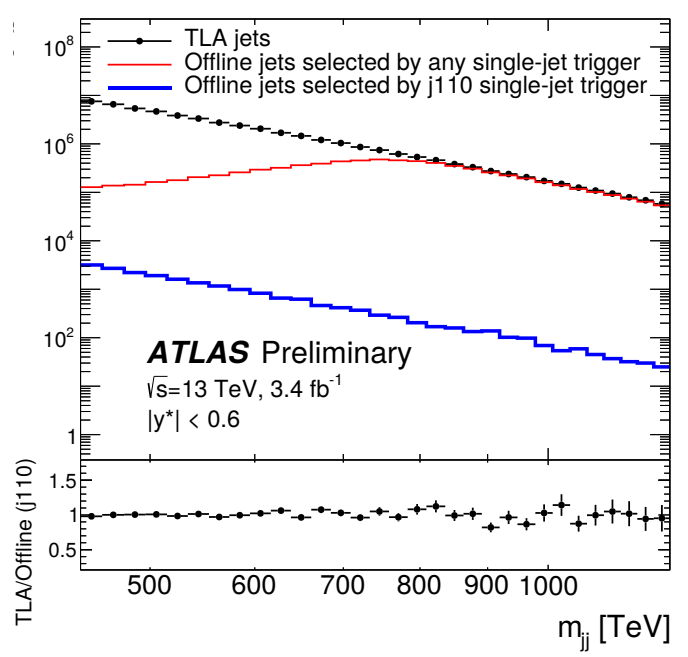

(a)

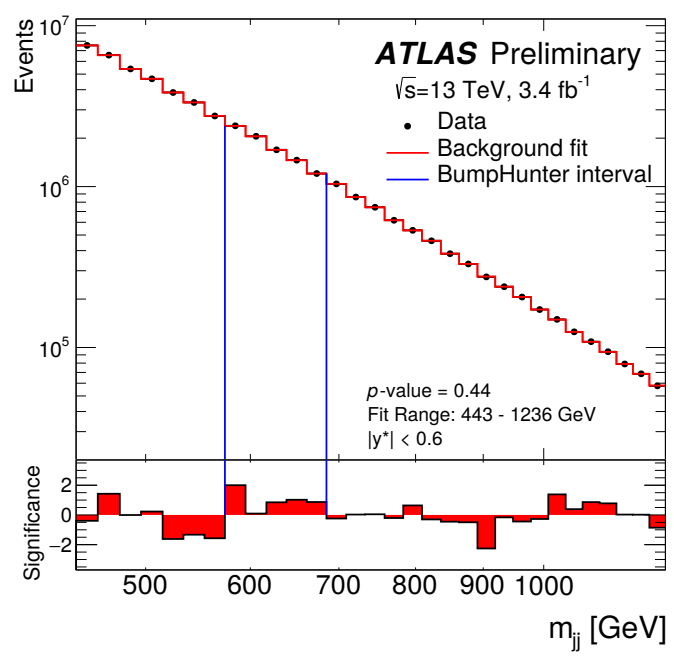

(b)

Figure 3: Dijet invariant mass comparison between the data used by the TLA and the data selected by using an OR of any single jet trigger for the search(a). The reconstructed dijet mass distribution for $\mathrm{y}^{*}<0.6$ (b).The lower panels indicate the significances of data-fit differences in bin-by-bin basis.

shows the benefit of unprescaled trigger in the TLA jets. The number of events below $800 \mathrm{GeV}$ are significantly increased. One can also see the agreement between the shape of $m_{j j}$ distribution between HLT and offline jets.

Most discrepant regions that are identified by the Bumphunter algorithm [3] lies between 574 and $685 \mathrm{GeV}$ as shown in Figure 3b. It has a p-value of 0.44 for $\mathrm{y}^{*}<0.6$ and 0.19 for $\mathrm{y}^{*}<0.3$. Hence, there is no significant excesses found. 
The observed upper limit on $m_{j j}$ on the cross-section, $\sigma$ times acceptance, $A$, times branching ratio, $B R$ of a leptophobic ZâĂŹ simplified model are shown in Figure 4b. Gaussian contributions of $Z^{\prime}$ signal ranging from $3 \mathrm{pb}$ at $450 \mathrm{GeV}$, to $9 \mathrm{pb}$ at $600 \mathrm{GeV}$, to $0.7 \mathrm{pb}$ at $850 \mathrm{GeV}$ are excluded with $95 \%$ credibility level. Figure 4 a shows the upper limits for the Z' model obtained from the

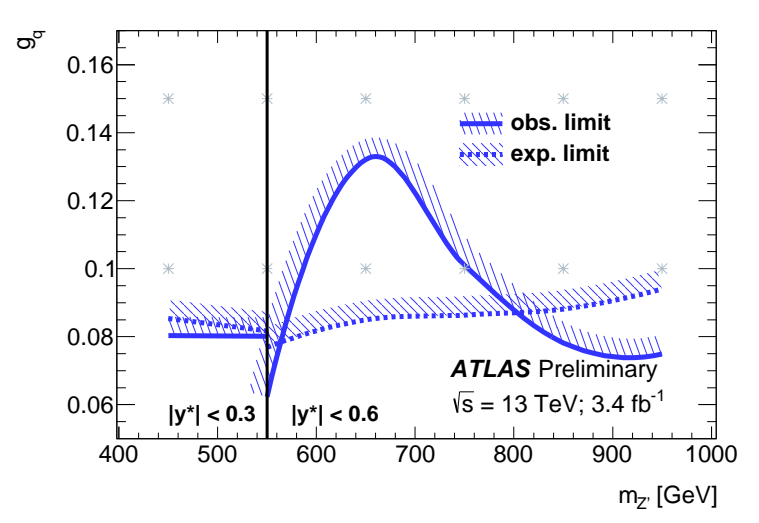

(a)

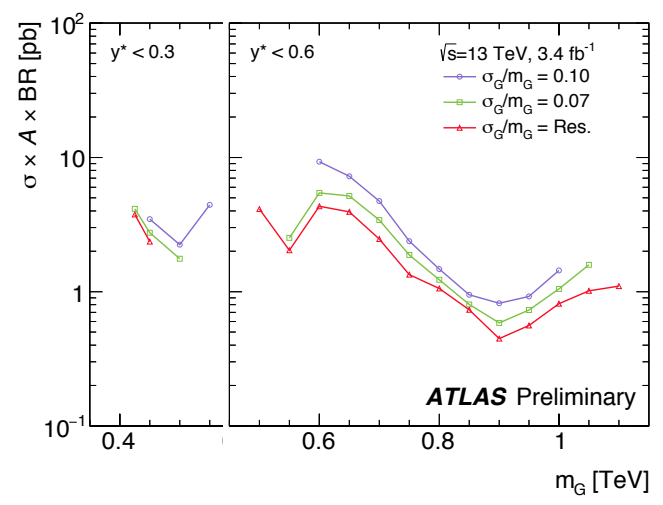

(b)

Figure 4: The ratio of observed limit to theoretical cross sections of Z' model for the TLA (a). Upper limit observed from the mjj on the effective cross sections are obtained for three different widths. A black vertical lines separated two signal regions(b).

$m_{j j}$ distribution plotted in a quark couplings, $g_{q}$, as a function of Z' mass, $m_{Z^{\prime}}$.

\section{References}

[1] Ricardo Abreu et al. Search for low-mass dijet resonances with Trigger-Level Analysis. ATLAS-CONF-2016-030. Geneva:CERN, Jun. 2016. [https://cds.cern.ch/record/2161135]

[2] ATLAS collaboration. Jet global sequential corrections with the ATLAS detector in proton-proton collisions at $\sqrt{s}=8 \mathrm{TeV}$. Tech. rep. ATLAS-CONF-2015-002. Geneva: CERN, Mar. 2015. [https://cds.cern.ch/record/2001682]

[3] G. Choudalakis. On hypothesis testing, trials factor, hypertests and the BumpHunter. arXiv:1101.0390 [physics.data-an]. 